\title{
Investigation of Traffic Accident Hotspots in Yazd City using GIS
}

\section{Ali Reza Tavakoli Mehr ${ }^{1} \mathbb{D}$, Mojtaba Salimi $^{2} \mathbb{D}$, Gholam Hossein Halvani $^{3} \mathbb{D}$, Mahmoudreza Peyravi $^{4} \mathbb{D}$, Hekmatollah Moradi $\mathbb{}$}

\author{
Date of submission: 22 Oct. $2020 \quad$ Date of acceptance: 20 Jan. 2021
}

\section{Original Article}

\begin{abstract}
INTRODUCTION: The importance of safety and prevention of traffic accidents have highlighted the necessity of research and investigation in this field. The present study aimed to identify and eliminate the risk factors underlying the occurrence of inner-city traffic accidents. In so doing, scientific and effective solutions can be provided at the lowest cost to implement the slogan of "prevention is better than cure".

METHODS: In this study, the accident rate-severity index method was used to identify traffic accident hotspots. The spatial units in which this index (I) was more than twice the average of the total spatial units were identified as Hot Zones

FINDINGS: In the present study, the study area (6,600 meters) was divided into 66 100-meter spatial units. Using the accident rate-severity method, $6(600 \mathrm{~m})$ and $19(1900 \mathrm{~m})$ spatial units were identified as Hot Zone (9.1\%) and Yellow Zone (28.8\%), respectively. Finally, 41 Cold Zone spatial units (62.1\%) were identified $(4,100 \mathrm{~m})$.

CONCLUSION: As evidenced by the obtained results, the following measures can be effective in the reduction of road accidents: retrospection of traffic signs in terms of number, size, location, height, installation of speed bumps in the Hot Zone and Yellow Zone spatial units, timely pruning of trees along the road-construction of underpasses and overpasses in some important points of the route (accident hotspots and Yellow Zone). Furthermore, the development of a comprehensive and long-term educational program to improve traffic safety culture in kindergartens and schools can be effective in reducing road accidents.

Keywords: Rate-Severity Index; Hot Zones; Spatial Unit
\end{abstract}

How to cite this article: Tavakoli Mehr AR, Halvani G, Moradi H, Ahmadi Marzaleh M. Investigation of Traffic Accident Hotspots in Yazd City using GIS. Sci J Rescue Relief 2021; 13(1): 42-48.

\section{Introduction}

oad accidents are on the rise across the globe since the development of transportation infrastructure is not in accordance with other sectors, such as industry and real estate. Therefore, traffic accidents are acknowledged as the leading cause of death and/or disease worldwide (ICMR 2009). These accidents often result in deaths, injuries, or casualties in people around the world. Traffic accidents are responsible for 24,000 deaths in Iran with a population of 77 million and 19 million registered cars and motorcycles (1).

The high social and economic costs of road accidents and their irreparable psychological effects on individuals and communities have highlighted the importance of accurate identification of the causes and prevention of accidents (2). One of the effective and necessary measures in this regard is the provision of various methods for the identification and prioritization of

1-Master Student, Occupational Health Engineering, School of Health, Shahid Sadoughi University of Medical Sciences and Health Services, Yazd, Iran

2-Department of Geography and Urban Planning, University of Tehran, Tehran, Iran

Assistant Professor, Department of Occupational Health Engineering, School of Health, Shahid Sadoughi University of Medical Sciences 3-and Health Services, Yazd, Iran

4-Department of Health in Disasters and Emergencies, School of Management and Medical Information, Shiraz University of Medical Sciences, Fars, Iran

Correspondence to: Hekmatollah Moradi, Email: morad2063@yahoo.com 
accident-prone places and the elimination of risk factors in these zones.

Due to budget and executive constraints, it is not possible to secure all accident-prone locations, and it is necessary to take the necessary measures with proper management to eliminate accidentprone places with higher priority (3). After cardiovascular diseases, traffic accidents are the second leading cause of death in the country, responsible for more than 20,000 deaths per year. Moreover, out of 190 countries, Iran has the highest accident deaths and injuries, immediately after Sierra Leone.

This position is not honorable at all; rather it is a sign of cultural lag or cultural backwardness as denoted by sociologists. One person is killed every 19 minutes, leaving a total of 23,000 deaths and 280,000 injuries each year in 800,000 accidents. This has led to the death of 500,000 people in the last 20 years (4). Loo BP was the first to introduce a model for identifying accident hotspots on road networks called Hot Zones in Hong Kong (5).

In the mentioned research, accident hotspots have been identified within city limits with different modeling. It was used to quantify the risk of road accidents and obtain the risk score of accident components, including severity (amount of damage, injury, and fatality) and recurrence of accidents in each spatial unit (using the method of accident rate-severity). Dilip Goswami et al. (2015) conducted a study entitled "Implication of GIS Technology in Accident Research in Bangladesh". This study used Hazardous Location Index analysis to identify accident-prone areas of Joydebpur-Jamuna Bridge National Highway (NH405).

The locations in the study area which were in urgent need of practical and environmental measures were identified using GIS techniques. It was found that about $11.50 \%$ of accidents occurred in only $2.63 \%$ of the total length of the highway (highly accident-prone); in addition, $12.41 \%$ length of the highway is accident-prone. In general, it was reported that $44.73 \%$ of all accidents occurred in $15.04 \%$ of the length of the highway.

Therefore, about $15.04 \%$ length of the highway needs counterstrike measures against accidents through road safety programs, and $84.96 \%$ of the highway is safe for travel (6). The results of the stated study revealed that about $65 \%$ of the road is passing over very high-risk areas, $25 \%$ over high, and $10 \%$ over mid-risk areas (7). Along the same lines, Mohammad Rahmani (2016) carried out a study entitled "Zoning of road accident-prone to determine the black spots using GIS (case study: Malayer-Hamedan road)."

The analytic results using GIS and mapping software to just point and mapped out a route feature in the form of functional maps. Finally, the Hamedan-Malayer route was zoned in fourspectrum, safe, relatively safe, dangerous, and accident-prone, and necessary measures were to reduce road accidents were presented (8). The main purpose of the current study was the analysis of inner-city traffic accidents using GIS.

The accident rate-severity index method has been used to identify accident hotspots. The importance of ensuring the safety and prevention of traffic accidents has highlighted the necessity of research and investigation in this area. The present study aimed to identify and eliminate the Risk Factors Underlying the Occurrence of innercity traffic accidents. In so doing, the scientific and practical solutions can be provided at the lowest cost to implement the slogan of "prevention is better than cure". The results of the present study can be optimally used in all urban roads of the province and the country.

\section{Methods}

This descriptive-analytical cross-sectional study assessed the hot zone and analyzed traffic accidents in the area of Shahid Beheshti Square to Abuzar Square in Yazd using Geographic Information System (GIS). The study population included all traffic accidents in the selected inner city in 2016. Accident hotspots were identified by different modelling of the Hot Zone method in the GIS environment $(9,10)$. To this end, the study population was one year of accidents occurred from Beheshti Square to Abuzar Square in Yazd with a length of approximately $14 \mathrm{~km}$ (round trip).

In this research, sampling was not performed, and all traffic accidents in the selected area were regarded as a sample. In the study area in 2016, 541 accidents were registered in the archives of the Traffic Department of Yazd. Nonetheless, due to the incompletion of the required information in 59 accidents, finally, 482 accidents were selected as the sample size. Among the methods of identifying accident hotspots in road accidents, according to the items of the research 
questionnaire and the volume of information provided by the Yazd Traffic Department, the accident rate-severity index method was employed to identify accident hotspots (Hot Zone).

The questions of this research included the following:

1. How many Hot Zone points are there in the study area?

2. How many medium-risk and low-risk points are there in the study area?

3. How are the central and dispersion indices of accident-prone spatial points in the study area?

4. What is the Risk priority number (RPN) of each Hot Zone?

5. Is there a relationship between the RPN of accident-prone points and the type of accidents?

6. Is there a relationship between the RPN of accident-prone points and the type of crashed vehicle?

7. Is there a relationship between the frequency of road accidents and the educational level of the victims?

8. Is there a relationship between the frequency of road accidents and the marital status of the victims?

9. Is there a relationship between the frequency of road accidents and the history of illness in the victims?

10. Is there a relationship between the frequency of road accidents and physical disability in people affected by the accident?

11. What is the priority of road accidents in terms of the cause of the accident?

The accident rate-severity index method: This method works similar to the Average Crash Frequency (ACF), except that the coefficient of severity is considered for different types of accidents based on the number and then according to their severity. Its applications are similar to the ACF Method (for small towns, an area in a large city, and intercity roads). The table of suggested coefficients used in the state of Georgia in the United States and in most countries was used in the current study to weigh the severity of accidents (fatal, injury, and damage) (Table 1).
Table 1. Proposed coefficients of the state of

Georgia for different types of accidents (11)

\begin{tabular}{cc|}
\hline Accident severity & Suggested ratio \\
\hline Fatal road accident & 9 \\
Injury road accident(Class A) & 4 \\
Injury road accident(Class B) & 3 \\
Injury road accident(Class C) & 2 \\
Damage-inflicting accidents & 1 \\
\hline
\end{tabular}

\section{Types of injuries}

Injury Accidents (Class A): It is an accident in which the injured people are hospitalized and at least one of them is likely to be disabled.

Injury road accident (Class B): In which the injured people are hospitalized without any possibility of disability.

Injury road accident (Class C): in which the injured are treated on an outpatient basis.

In this method, for each position (spatial unit), a special index is calculated using the following formula:

$I=9 \times T_{f}+4 \times T_{i a}+3 \times T_{i b}+2 \times T_{i c}+T_{d}$

In the above formula:

I: The accident rate-severity index in Basic Spatial Unit (BSU

$\mathrm{T}_{\mathrm{f}}$ : Fatal accidents (per BSU)

$\mathrm{T}_{\mathrm{ia}}$ : Injury road accidents (Class A) per BSU

$\mathrm{T}_{\mathrm{ib}}$ : Injury road accidents (Class B) per BSU

$\mathrm{T}_{\mathrm{ic}}$ : Injury road accidents (Class $\mathrm{C}$ ) per BSU

$\mathrm{T}_{\mathrm{d}}$ : Damage-inflicting road accidents per BSU

Identification of traffic accident hotspots in BSUs using accident rate-severity index method

The spatial units in which the accident rateseverity index (I) is more than twice the average of the total spatial units are identified as Hot Zones (11).

\section{Findings}

As evidenced by the obtained results, in 2016, 482 accidents occurred in the study area (Beheshti Square to Abuzar Square in Yazd with a length of 6,600 meters). In the current study, the study area was divided into 66 100-meter spatial units. Moreover, 6 Hot Zone points and 19 Yellow Zone points were identified using the accident rateseverity index. The maps related to Yazd, selected 
route, spatial units, and location map of important offices and centers in the study area are presented in Figures 1-3.

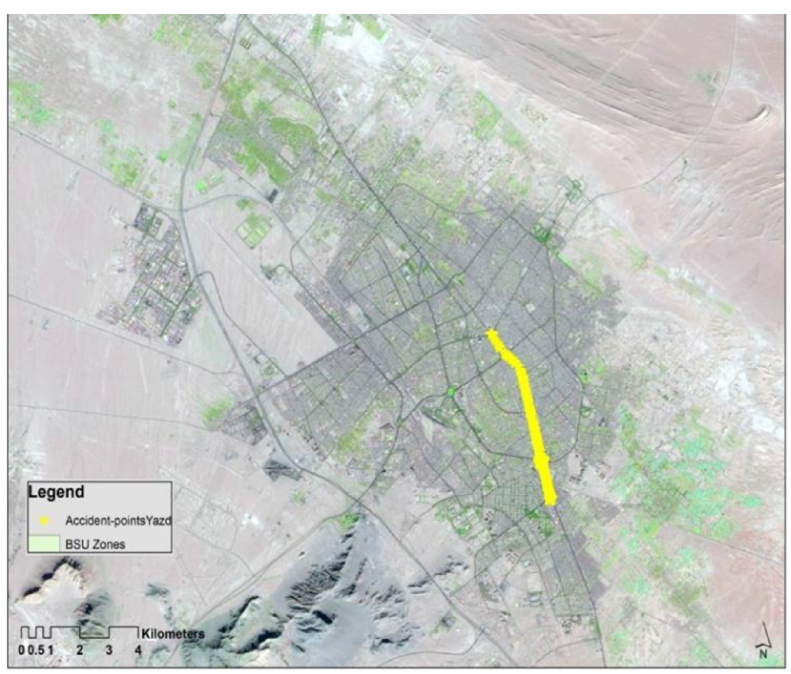

Figure 1. Map of Yazd and the study area

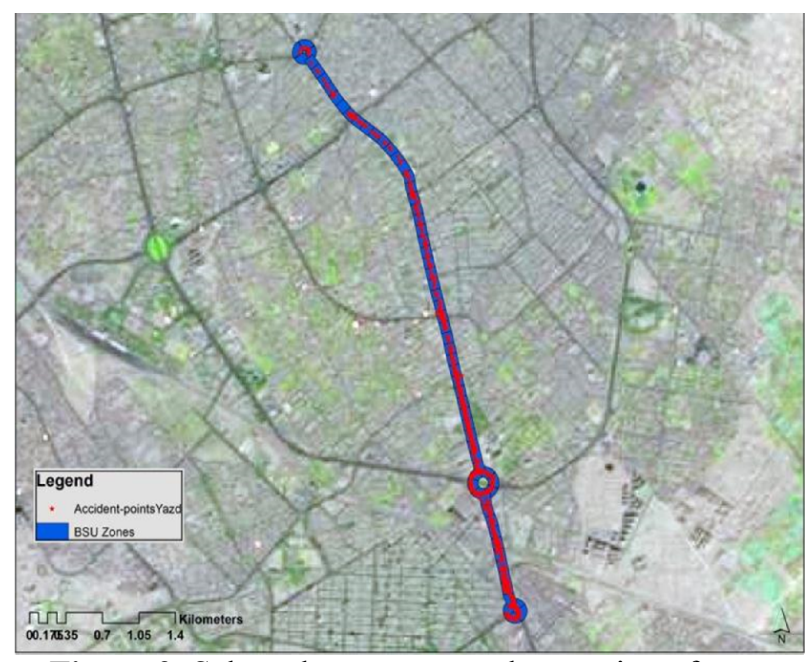

Figure 2. Selected route map and scattering of events

Zoning of spatial units based on the accident rateseverity index: A total of 66 spatial units were examined in the present study. After quantifying the accident risk by the accident rate-severity method, 41(62.1\%) spatial units (4,100 meters) were identified as Cold Zones, and 19 (28.8\%) spatial units were identified as Yellow Zone (1900 $\mathrm{m})$. These spatial units are potential accident hotspots and they should be given the second priority after accident hotspots. Finally, 6 space units (600 meters) were identified as Hot Zones (9.1\%) (Table 2) (Figure 4).

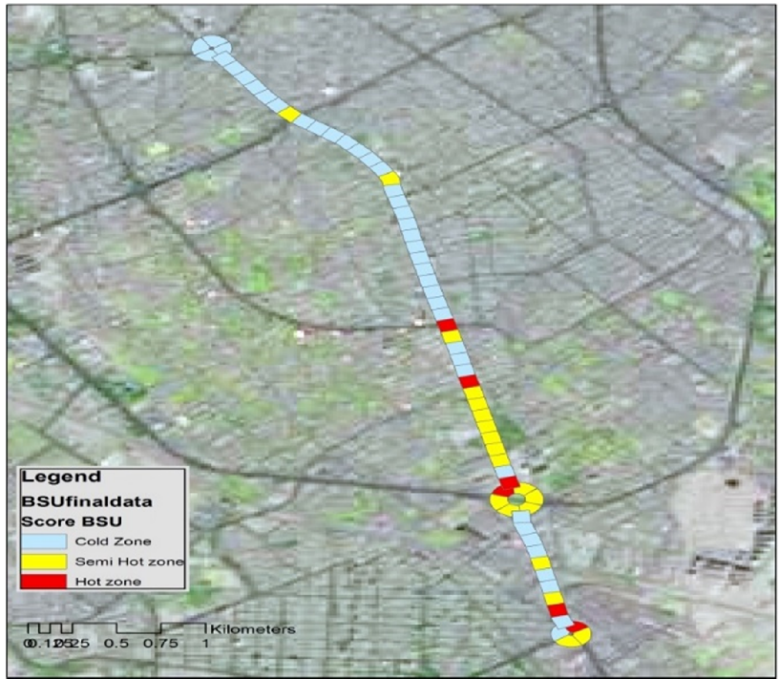

Figure 3. Hot Zones of Yazd

zone
吕
至

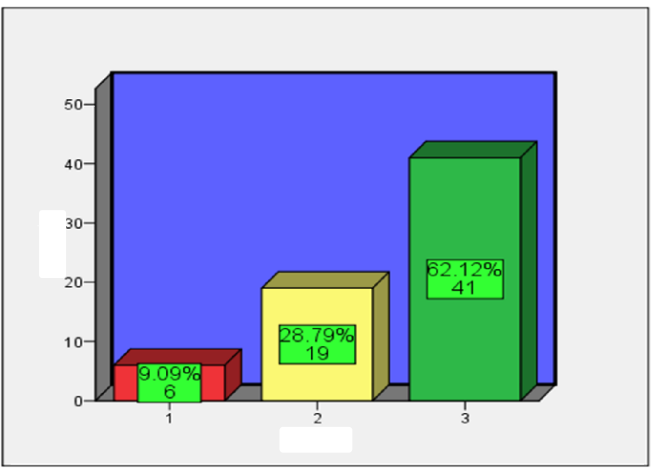

Figure 4. Spatial frequency based on the accident rate-severity index

Table 2. Frequency of spatial units based on the accident rate-severity index

Variable Frequency Percentage Rank

\begin{tabular}{ccccc} 
& Hot Zone & 6 & 9.1 & 3 \\
Special & Yellow & 19 & 28.8 & 2 \\
units(BSUs) & Zone & & & \\
\cline { 2 - 4 } & Cold Zone & 41 & 62.1 & 1 \\
Total & 66 & 100 & \\
\hline
\end{tabular}

\section{Discussion and Conclusion}

The present study aimed to identify Hot Zones in the area of Beheshti Square to Abuzar Square in Yazd in 2016 using the rate-severity index (I) method to quantify the risk in the GIS environment. To determine the accident-prone zones, the accident rate (AR) for each BSU was selected based on the rate-severity index method. 
All spatial units with a risk index of greater than twice the average were selected as Hot Zones.

The mean distance and twice the mean were selected as a Yellow Zone. Spatial units with a below-average risk index were identified in the Cold zone. Out of 482 accidents in the study area, the highest frequency of accidents was related to damage-inflicting ones $(434 ; 90 \%)$. The total frequency of injury accidents, regardless of the type of zoning, was reported as $45(9.4 \%)$ cases. The injury accidents were classified based on the coefficient table proposed by the state of Georgia (Class A, B, and C injury events).

Class $\mathrm{C}$ injury events ranked the second with a frequency of $31(6.4 \%)$ cases. Class A and B injury events stood in third place with a frequency of 7(1.5\%) cases. Of all the studied accidents, 3 (6 $\%)$ fatal accidents were registered. Hoshyar et al. (2017) conducted a study entitled " Spatial analysis of intra-city accidents (Case study: Urmia city". The results of the mentioned study indicated that out of 5,785 accidents in 2012, damageinflicting accidents with a frequency of 3584 $(62 \%)$ cases were the most common types of accidents in the city.

In terms of frequency, injury accidents with $2,171(37.5 \%)$ cases and fatal accidents with 30 $(0.5 \%)$ cases ranked second and third. In the assessment of accidents in Urmia in $2013(5,846$ cases), damage-inflicting accidents stood in the first place with $3,333(57 \%)$ cases. Thereafter, injury accidents and fatal accidents ranked second and third with 2,483(42.5\%) and $30(0.5 \%)$ cases, respectively (12).

The results of the study are consistent considering the differences between intra-city and inter-city driving styles (high traffic load and low driving speed on inner-city roads). After the quantification of accident risk by the rate-severity method, 6 spatial units (600 meters) from the study area were identified as Hot Zones $(9.09 \%)$. A number of $19(28.78 \%)$ spatial units $(1900 \mathrm{~m})$ were identified as Yellow Zone (a spatial unit that was identified as a potentially dangerous location with a lower risk, compared to the Hot Zone using different models and methods). This zone is the potential to convert to Hot Zone spatial units.

The other spatial units $(n=41)$ with a length of 4, 100 meters were identified as Cold Zone) (62.12\%). Mohammad Rahmani (2016) carried out a study entitled " Zoning of road accidentprone to determine the black spots using GIS (case study: Malayer-Hamedan road)". In the referred study, the Hamedan-Malayer route was zoned in four-spectrum, safe, relatively safe, dangerous, and accident-prone, and necessary measures were presented to reduce road accidents (8).

From the beginning of Beheshti Square to Fatemiyeh crossroads, due to the installation of a plastic guard rail in the center of the street, there is no possibility of unauthorized overtaking, overtaking violation, and the occurrence of frontal accidents. In this regard, the central guard rail along the entire route can be used as one of the ways to reduce the occurrence of accidents in the study area.

In the study area, Fatemiyeh crossroads was one of the places with the highest number of accidents. In this crossroads, despite the installation of the "No U-Turn" sign, high traffic load, and the unfamiliarity of drivers and passengers to go to the city center lead to accidents in most cases. To reduce accidents in this area in the short term, it may be helpful to install a sufficient number of large flashing "No U-Turn" signs within a driver's field of vision.

Moreover, during rush hours, the presence of traffic police officers in the mentioned place to guide people and prevent violations can provide the ground for the reduction of accidents at the mentioned crossroads. Furthermore, in a longterm plan, the construction of underpasses and overpasses can be on the agenda. Shohada-ye Mehrab Square was one of the places with a large number of accidents and high traffic load. A field study revealed that one of the major causes is the proximity of 7th Tir Park to this square.

Most of the travelers and patients' companions admitted to Mortaz, Bahman, and Mojibian hospitals set up tents and stay in this park, especially in summer. This has led to a high traffic load in this square and the surrounding area towards 7th Tir Park, which in turn, has resulted in the occurrence of numerous traffic accidents in this area. Two Hot Zone spatial units and five Yellow Zone spatial units were identified in the area of Shohada-ye Mehrab Square.

Moreover, seven Yellow Zone spatial units were recognized in a row in the area of 7th Tir Park as potential Hot Zone spatial units. In order to reduce the traffic load and accidents in this area in the short term, we can prohibit setting up tents in 7th Tir Park. In addition, the authorities should 
consider other places for passengers and companions of hospitalized patients. In the long run, diminishing the number of hospitals in this area can be a good idea to reduce the traffic load and accidents.

During the field study (latitude and longitude of the crash site-checking the location of traffic signs and the distance from speed bumps to accident site), 12 accidents were observed in the study area, confirming the high traffic load in this area. In addition, most of the observed accidents due to the nature of the accidents which were minor damages were resolved with the agreement of the parties without registration and informing the responsible departments.

Traffic accident statistics exceed the registered cases, and only the registered cases were used to identify accident hotspots in the present study. Finally, six traffic accident spatial units were identified. For more accurate and comprehensive identification of the number of traffic accident hotspots (there is the possibility of converting Cold Zone into Yellow Zone and Yellow Zone into Hot Zone), drivers can be required to refer to the traffic police by passing and enforcing the law in this regard.

In some cases, minor accidents cause high traffic and increase the risk of further accidents. One of the main causes of inner-city traffic is other drivers' stop to watch and sometimes film. In some other cases, when accidents lead to injuries, this behavior of drivers delays the arrival of ambulances and rescue forces. Apart from culture-building in the long run, the imposition of fines on these drivers who stop just to watch an accident can be proposed as a short-term suggestion to reduce the risk of traffic accidents.

To prevent traffic accidents, apart from the annual technical inspection of cars, checking brake pads should be a priority for drivers on a monthly or at least seasonal basis (Observing a chain accident during data collection). Helmets and having a motorcyclist license are a compulsion and a law, and special attention to its implementation by traffic police officers can help reduce traffic accident injuries (45 injury accidents) in the study area and the whole city. Lack of speed bumps in the whole route and lack of central guard rails in the most part of the route has paved the ground for unauthorized overtaking. The installation of the mentioned devices in the
Hot-Yellow Zone spatial units can be considered a solution for the reduction of traffic accident

As a safety point for field data collection in similar cases in future research, due to the dangerous nature of the task of collecting traffic accident hotspots (locating accident sites with GPS), there is a possibility of accidents for researchers. Responding to people, dealing with businessmen, marketers, police, and even drivers cause disturbances and interruptions in various cases. Safety measures taken in collecting this information include wearing appropriate and comfortable clothes with yellow bands (fluorescence) and the involvement of two people in data collection.

One person locates with a GPS, while the other one registers information and inspects the passing vehicles to inform the first person. Meanwhile, in the present research, the entire route of about $6 \mathrm{~km}$ was walked several times by the researcher for data collection (about $60 \mathrm{~km}$ ). In the future similar studies with larger study areas, it will pose a serious challenge to the researcher.

It is suggested that in future studies, the traffic officer locate and record the longitude and latitude of accident sites in accident investigation form using a GPS device. The nature of road accidents (suburban) is completely different from traffic accidents (inner city). In road accidents, due to the high speed of vehicles, one mistake can be the last mistake of the driver, and the majority of accidents are injury and fatality. Nonetheless, in traffic accidents, due to the traffic load of the inner city road and lower speed, the accidents are mainly damage-inflicting.

\section{Acknowledgments}

None

\section{Conflict of Interests}

Authors have no conflict of interests.

\section{References}

1. Shafabakhsh GA, Famili A, Bahadori MS. GISbased spatial analysis of urban traffic accidents: case study in Mashhad, Iran. J Traffic Transport Eng 2017; 4(3): 290-9 (In Persian).

2. Durduran SS. A decision making system to automatic recognize of traffic accidents on the basis of a GIS platform. Expert Syst Appl 2010; 37(12): 7729-36. 
3. Iyanda AE. Geographic analysis of road accident severity index in Nigeria. Int J Inj Control Saf Prom 2019; 26(1): 72-81.

4. Khani F, Samsam Shariat S, Atashpour S. Study of the relationship between personality traits with occupational accidents and quality of sleep among road drivers in Isfahan city (Year 1390). Strategic Res Soc Problems Iran Univ Isfahan 2013; 1(4): 75-88 (In Persian).

5. Loo BP. The identification of hazardous road locations: a comparison of the blacksite and hot zone methodologies in Hong Kong. Int $\mathrm{J}$ Sustainable Transport 2009; 3(3): 187-202.

6. Goswami D, Morshed MM, Hasan MS. Implication of GIS technology in accident research in Bangladesh. $\mathrm{J}$ Bangladesh Instit Plan 2015; 8: 159-66.

7. Zeller KA, Wattles DW, DeStefano S. Incorporating road crossing data into vehicle collision risk models for moose (Alces americanus) in Massachusetts, USA. Environ Manag 2018; 62(3): 518-28.
8. Rahmani M. Zoning of road accident-prone with determine the black spots by using GIS (case study: malayer-hamedan road). Environ Based Territorial Plan 2016; 9(34): 155-75 (In Persian).

9. Kavousi A, Moradi A, Rahmani K, Zeini S, Ameri P. Geographical distribution of at fault drivers involved in fatal traffic collisions in Tehran, Iran. Epidemiol Health 2020; 42: e2020002 (In Persian).

10. Meeker J, Perry A, Dolan C, Emary C, Golden K, Abla C, et al. Development of a competency framework for the nutrition in emergencies sector. Public Health Nutr 2014; 17(3): 689-99.

11. AFRC Engineering. Criteria for determining the incident points of the city. Tehran: Transportation and Traffic Department of Tehran Municipality; 2012.

12. Hoshyar H, Sharifi B. Spatial analysis of intra-city accidents (Case study: Uromia city). J Geographical Eng Territory 2017; 1(1): 90-101 (In Persian). 